\title{
Experimental Study on a New-type of Water Mist Extinguishing Nozzle
}

\author{
Zhizhong Zhang \\ School of Energy and Environment, Xihua University \\ Chengdu 610039, China \\ 653452406@qq.com
}

\author{
Qinggang Li \\ School of Energy and Environment, Xihua University \\ Chengdu 610039, China \\ lqg@mail.xhu.edu.cn
}

\begin{abstract}
Base on the study and analysis of existing ordinary nozzle and their atomization mechanism, the New-type of water mist fire extinguishing nozzle is developed and experimentally studied with Laser Doppler Velocimetry (LDV) and Phase Doppler Particle Analyzer (PDPA). The characteristics of flow coefficient $K$, atomizing angle $\theta$, Sauter Mean Diameter (SMD) and the distribution of axial velocity in different test pressure are investigated. We draw the conclusion that the best range of working pressure is 1.5 MPa to 3.0 MPa, SMD is similar to "V" shape, axial velocity is similar to normal distribution, the average of flow coefficient $K$ is $\mathbf{1 9 5 5 . 5 7 5}$ and the range of atomizing angle is $60^{\circ}$ to $62.8^{\circ}$.
\end{abstract}

Keywords-water mist; SMD; axial velocity; atomizing angle

\section{INTRODUCTION}

Water mist fire extinguishing technique, with its high efficiency, environmental protection, energy saving, has become main fire extinguishing technology. It is one of the hottest spot in the research of fire science, and water mist fire extinguishing technology at home and abroad has made significant progress ${ }^{[1-2]}$.

However, water mist fire extinguishing sprinkler is the key part of water mist fire extinguishing system, the traditional atomizing nozzle atomization effect is not ideal. Atomizing angle and protection radius are small, which limits the fine water mist fire extinguishing system application scope. At present, there are many methods to solve such problems as atomizing angle, protection radius and spray momentum, including combined atomization nozzle, rotary spray nozzle, and medium atomization nozzle. These methods are widely used, because the atomization effect is good, and processing production is convenient.

In view of the deficiency of the traditional direct spray nozzle, the job designs a new type of the water mist fire extinguishing sprinkler, and provides a new nozzle water structure, whose characteristic is that inlet channel axis which is arranged beside the center inlet channel axis is particular equal angle, and spins in the same direction.

Due to the complexity of turbulent motion and atomization mechanism, researchers should have solid fluid mechanics and mathematics knowledge to establish the physical and mathematical model. At present, there isn't a set of perfect nozzle design calculation procedure, therefore, the experiment research plays a very important role in fine water mist fire extinguishing sprinkler design manufacturing process ${ }^{[3-4]}$. The main tasks of the experiment are to measure the atomization performance parameters of water mist fire extinguishing sprinkler and to evaluate the fire extinguishing performance. Experiment results show that the atomizing nozzle, with different structure from the traditional direct nozzle, is more suitable for low voltage condition.

\section{EXPERIMENT SYSTEM AND METHOD}

\section{A. Experiment system}

Nozzle atomizing characteristic measuring work is tested on cold characteristic test stage, and the nozzle atomizing characteristics test system is shown in figure 1 .

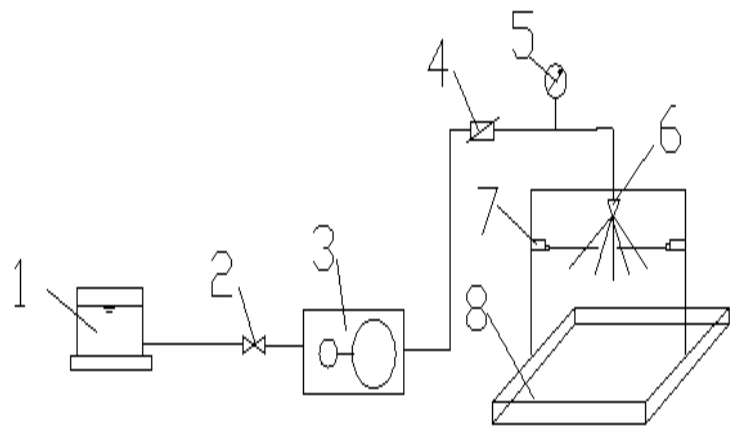

Fig.1 The experiment system

1 - Water supply box; 1 - valves; 3 - pressure pump; 4 meter;

5 - Pressure gauge 6 - experiment nozzle; 7 - LDV; 8 organic glass set tank

Test bench is composed of water supply system, spray system and test system. Supply pressure can be adjusted in a range of $0 \sim 4.0 \mathrm{MPa}$. The test system is composed of flow measurement, pressure measurement, LDV, recording and processing data computer. And the spray system is composed of experiment nozzle and organic glass container.

At the beginning of the test, we make nozzle reach the predetermined condition through regulating pressure pump. The spray atomizing angle will be identified by the photo taken by digital camera through further processing. We calculate the flow characteristic coefficient $\mathrm{K}$ by the rate of flow $\mathrm{Q}$ which is 
get from the flow meter, and then measure the SMD and particle axial velocity through LDV in the fog field cross section.

\section{B. Nozzle structure}

As the figure 2 shows, the nozzle is composed of water inlet, mixing chamber and outlet and inlet including center inlet channel and lateral inlet channel. The character of the nozzle is that the lateral inlet channel axial line and hybrid cavity radial line form vertical intersection. The side inlet channels are located at the radial surrounding of the center inlet channel and settled in symmetric configuration. It is an angle of $30^{\circ}$ formed by the side inlet channel axial line , the intersected hybrid cavity radial line and center inlet channel axial line.

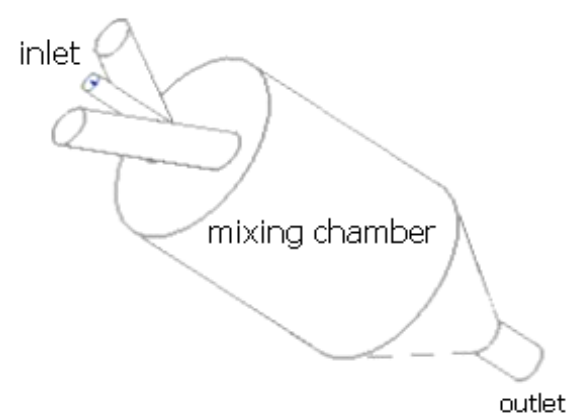

Fig.2 Internal structure of extinguishing nozzle

Nozzle has the side inlet channels which form a particular angle with the center inlet channel and center inlet channel axis, and the side inlet channels have equal angle and consistent spin. The side inlet channel spiral flow mixed with the direct current which produces axial kinetic energy, forming a vortex movement by the strong hybrid of central inlet channel direct flow and lateral inlet channel in the mixing chamber. Pushed by the center inlet channel axial kinetic energy, the mixed cyclone finally eject from the outlet of the sprinkler head, producing a strong collision with the atmospheric produce.

This structure decreases the side channel axial momentum loss to the lowest while maintaining a strong vortex. Compared with the traditional atomizing nozzle, its advantages are that owning bigger axial momentum, making fog drop through fire space to the utmost, realizing depth out fire, keeping the radial momentum of the water mist, increasing the atomizing angle of the water mist, expanding the out fire scope of water mist, as well as, small volume, simple structure, easy processing, low manufacturing cost.

\section{Droplet diameter measurement}

SMD to measure the position is perpendicular to the water mist sprinkler center axis and is located in the shower nozzle 1 $\mathrm{m}$ under the plane inside. The 24 marks in figure 3 are using the LDV, LDV and PDPA are used to measure droplet diameter $^{[5]}$. The FLOWSIZER software is the integrated platform of PDPA/LDV system hardware control, data acquisition and post-processing analysis, including many digital signals processing of advanced technology and providing the Doppler signal processing methods of analysis.

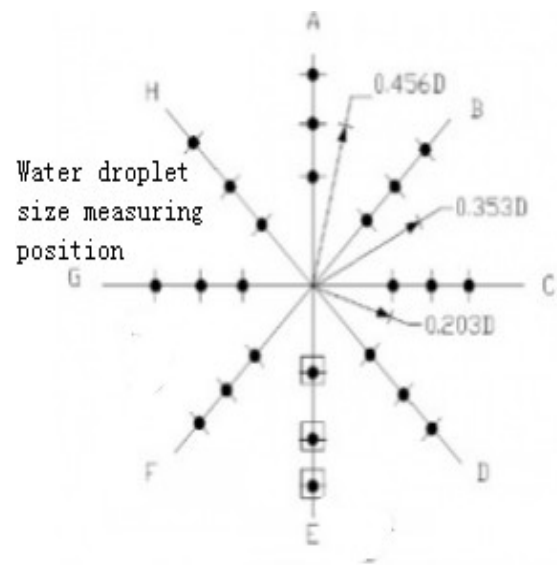

Fig.3 Droplet diameter measuring position

As figure 4 shows, through the PDPA test, the experiment can get the following distributing figures: the droplet axial velocity real-time figure, droplet diameter real-time figure, droplet diameter column figure, droplet axial velocity column figure, SMD and particle axial velocity, etc.

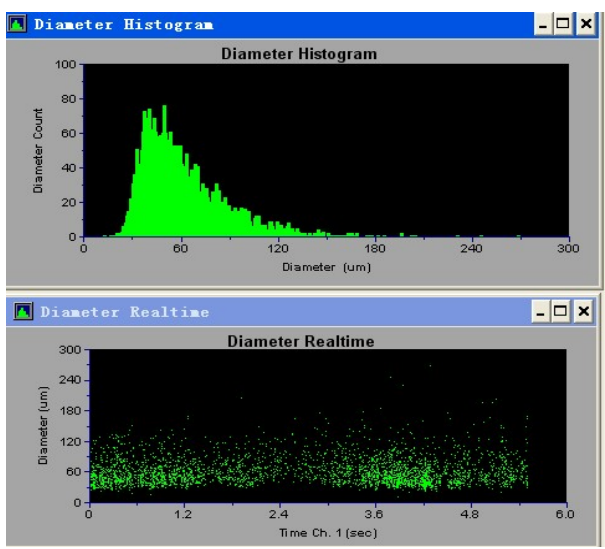

Fig.4 PDPA test data interface diagram

\section{THE EXPERIMENTAL RESULTS AND DATA}

A. Characteristics of flow coefficient $K$ and the test pressure

Flowing characteristic coefficient $\mathrm{K}$ computation formula is as follows ${ }^{[6]}$ :

$$
K=\frac{Q}{\sqrt{10 P}}
$$

Type:

$\mathrm{P}$ - Fine water mist sprayer entrance pressure, the unit is mega Pascal (MPa)

\section{$\mathrm{Q}$ - Flow, the unit is liter per minute $(\mathrm{L} / \mathrm{min})$}

The nozzle flow characteristic reaction of volume (or weight) flows with the changes of pressure value, it determines the capability of the water mist extinguishing nozzle.

As the figure 5 shows, in the four kinds of test pressure, the flowing coefficient measurement test, we get a fine spray nozzle flow coefficient $\mathrm{K}$ average, which was 1955.575 , and the nominal flow coefficient of the relative deviation is less 
than $5 \%$. Flow would have increased as the pressure increases, but limited to the nozzle structure size, the flow characteristics will gradually decrease.

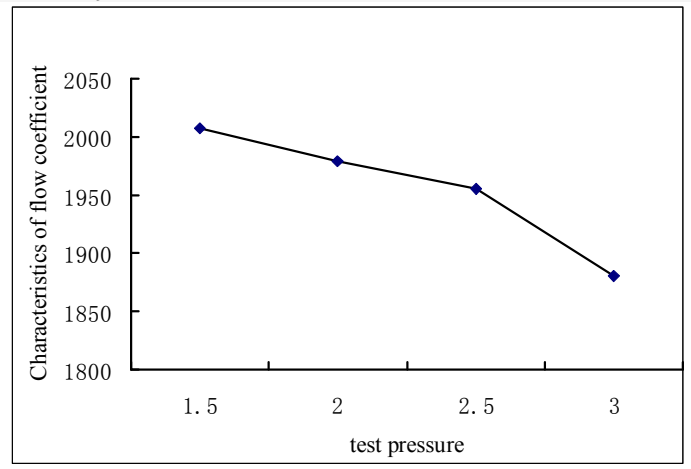

Fig.5 Characteristics of flow coefficient $\mathrm{K}$ and the test pressure

\section{B. Atomizing angle $\theta$ and test pressure}

Atomizing angle is representation spray expansion degree of quantity, in the nozzle exit for spray boundary of the tangent, two tangents of the angle which are export atomizing angle ${ }^{[7-8]}$. This experiment uses a digital camera to take pictures, and picture processing can get atomizing angle.

The size of the atomizing angle mainly depends on the liquid viscosity and surface tension, as well as the fluidic turbulence degree. Jet turbulence degree of increase will increase jet radial and axial velocity component ratio, urge the spray cone Angle bigger. At the same time, the liquid jet into gas environment pressure becomes back pressure, the injection pressure and back pressure difference $\Delta p l$ influence, high speed spray jet action may cause environmental gas generation strong disturbance, and make the atomizing angle of narrow, along with the increase of the pressure difference $\Delta p l$ and jet velocity further increase, this influence will become larger and larger.

The figure 6 can show that, atomizing angle $\theta$ with test pressure change also can produce change, with the test pressure $1.5 \mathrm{MPa}$, the atomizing angle maximum $62.8^{\circ}$, along with the increase of the test pressure, atomizing angle $\theta$ shows a trend of decrease, but change amplitude is not very big, on the whole, mixed cyclone water mist sprinkler atomizing angle $\theta$ is bigger, spray radius is bigger, covering a wider range.

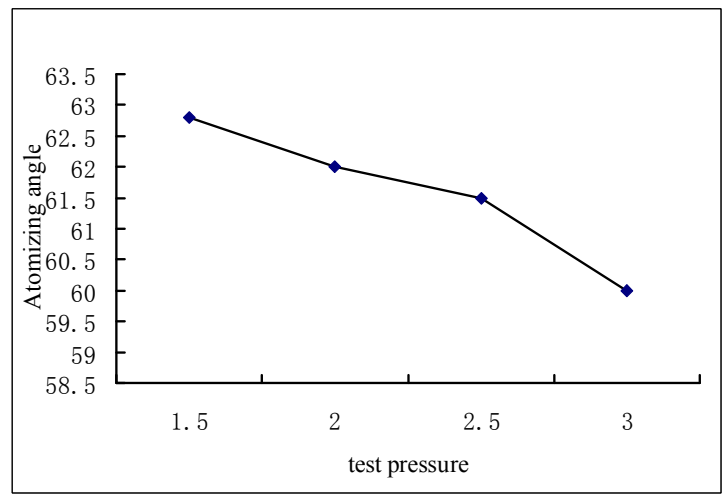

Fig.6 Atomizing angle and test pressure

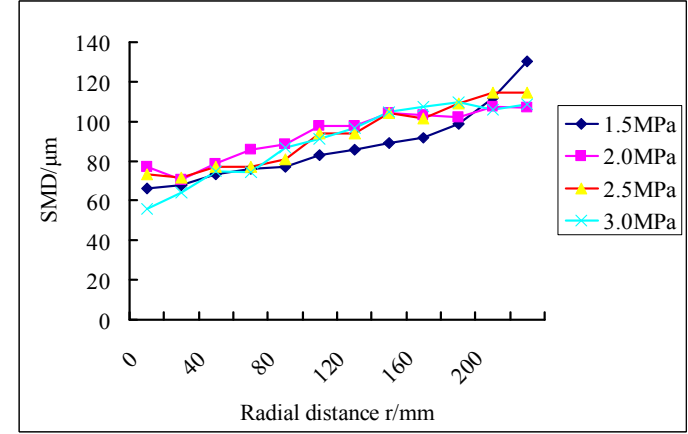

Fig.7 SMD and radial distance in different pressure

\section{SMD and the radial distance $r$ in different test pressure}

The experiment measuring path along the spray section center line (including central point and boundary point) is located in the shower nozzle $1 \mathrm{~m}$ under the in-plane, measuring step $20 \mathrm{~mm}$. Each test point for sampling 5000 particles, test data provides the section center's online SMD, as is shown in figure 7, the smallest droplet in spray central location is relatively thin, and SMD is the minimum .when the center point distance increases, SMD will increase, In other words, more to the edge, SMD is greater. Perpendicular to the central axis of water mist nozzle and nozzle located at $1 \mathrm{~m}$ as the origin, the SMD from origin to edge is similar to "V" distribution In 3.0 MPa, SMD minimum is $59.76 \mu \mathrm{m}$.

Back pressure of spray droplet size effect, the average particle size increases with back pressure and, reaches a maximum, which will slow down.

\section{Axial velocity and radial distance $r$ in different test pressure}

Nozzle of the axial velocity and SMD is the same pace, the figure 8 can show, the similarity and perpendicularity to the water mist sprinkler center axis is located in the shower nozzle in $1 \mathrm{~m}$ for the origin, the axial velocity is the largest, pressure for 3.0 $\mathrm{MPa}$ reaches the maximum axial velocity, the axial velocity from point of origin to the brink is similar to normal distribution.

In the test pressure for $3.0 \mathrm{MPa}$, axial maximum speed is $19.7686 \mathrm{~m} / \mathrm{s}$, and has a far penetration distance. The overall effect is good.

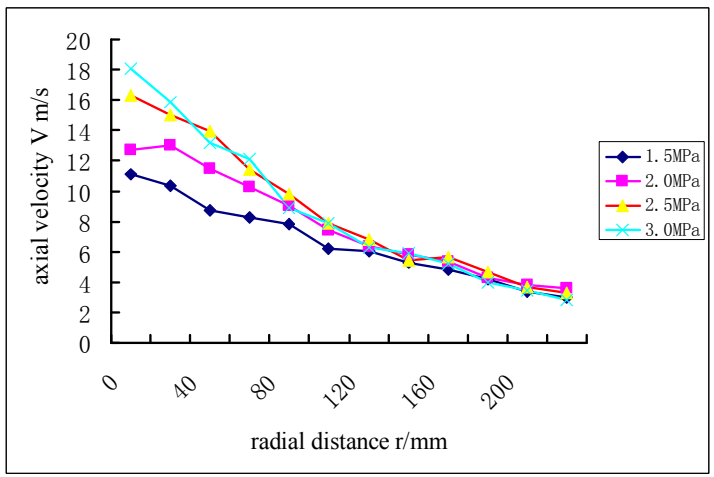

Fig.8 Axial velocity and radial distance in different test pressures 


\section{CONCLUSIONS}

1) In four kinds of test pressure, flow characteristic coefficient $\mathrm{K}$ decreases with pressure increase and the job design belongs to a small flow nozzle. To achieve its requirements of extinguishing fire, it is possible to get the optimal structure parameters by changing the nozzle inlet and outlet size to achieve the change of flow.

2) When the test pressure changes in $1.5 \mathrm{MPa}$ to $3.0 \mathrm{MPa}$ time, atomizing angle $\theta$ changes in $62.8^{\circ} \sim 60^{\circ}$, as well as pressure is $1.5 \mathrm{MPa}$, atomizing angle maximum $62.8^{\circ}$, atomization effects best.

3) In any kind of test pressure, when spray transverse section SMD is similar to the "V" shape, at the same point under different pressure, as pressure increases, SMD reduces. At the same point in 3.0 MPa pressure, SMD is the minimum.

4) The axial velocity from point of origin to the brink is similar to normal distribution. At the same point under different pressure, as pressure increases, the axial velocity will increase. when the test pressure under $3.0 \mathrm{MPa}$, the performance of the axial momentum is the maximum.

5) From the experiment, the work water mist fire extinguishing sprinkler has the larger axial momentum and radial momentum and is helpful for deep fire extinguishing and expanding the range of extinguishing fire. What's more, it has small volume, simple structure. The experiments shows that in $1.5 \mathrm{MPa} \leqq \mathrm{P} \leqq 3.0 \mathrm{MPa}$ medium voltage range, sprinkler atomization performs well, and kinetic energy index is good. It is a kind of the superior performance of nozzle and has broad prospect of application.

\section{REFERENCES}

[1] Guanghua He, Zhili Zhou, Yongjun Gong, Machine Tool and Hydraulics, Vol.36(2008),pp.80-82. (In chinese)

[2] Lijun Yang, Guobiao Cai, Zebao Hu, etc. Fire Science and Technology, Vol.21(2002),pp. 33-35.. (In chinese)

[3] Fang $\mathrm{Xu}$, Dong Wei, Qiang Liang. Fire Science and Technology,Vol.29(2010),pp.588-590. (In chinese)

[4] Jianming Cao: Spray. China Machine Press, Beijing, pp.1-79, 2005. (In chinese)

[5] GB/T 26785-2011, General technical specifications for water mist extinguishing systems and components .2011. (In chinese)

[6] Xin Cai, Xishi Wang, Guangxun Liao. Journal of Disaster Prevention and Mitigation Engineering, Vol.32(2012),pp.236-237. (In chinese)

[7] Junhu Zhou, Hualin Zhou, Weijun Yang, The Chinese Journal of Process Engineering, Vol, 7(2007),pp.652-655. (In chinese)

[8] Lingyu Hou, Xiaochun Hou: Nozzle Technology Handbook .China Petrochemical Press, Beijing, pp.66-70, 2002. (In chinese) 